\title{
Circuit
}

Musiques contemporaines

\section{Introduction : une SMCQ aux tiroirs}

\section{Maxime McKinley}

Volume 27, numéro 2, 2017

Souvenirs du futur : pour les 50 ans de la SMCQ

URI : https://id.erudit.org/iderudit/1040873ar

DOI : https://doi.org/10.7202/1040873ar

Aller au sommaire du numéro

\section{Éditeur(s)}

Circuit, musiques contemporaines

ISSN

1183-1693 (imprimé)

1488-9692 (numérique)

Découvrir la revue

Citer ce document

McKinley, M. (2017). Introduction : une SMCQ aux tiroirs. Circuit, 27(2), 5-9. https://doi.org/10.7202/1040873ar

Ce document est protégé par la loi sur le droit d'auteur. L'utilisation des services d'Érudit (y compris la reproduction) est assujettie à sa politique d'utilisation que vous pouvez consulter en ligne.

https://apropos.erudit.org/fr/usagers/politique-dutilisation/
Cet article est diffusé et préservé par Érudit.

Érudit est un consortium interuniversitaire sans but lucratif composé de l'Université de Montréal, l'Université Laval et l'Université du Québec à Montréal. Il a pour mission la promotion et la valorisation de la recherche. https://www.erudit.org/fr/ 


\section{Introduction : une SMCQ aux tiroirs}

Maxime McKinley

\begin{abstract}
La merveilleuse aventure de la Société de musique contemporaine du
Québec (SMCQ) : sa conception en 1965 avec Wilfrid Pelletier, Jean Papineau-Couture et Hugh Davidson; sa gestation de plus de neuf mois - car ce n'était pas un enfant ordinaire - et l'accouchement du 15 décembre 1966, avec les premiers cris, bouléziens et autres, sous la direction de Serge Garant.
\end{abstract}

- Maryvonne Kendergi ${ }^{1}$

Dans le cadre d'un numéro soulignant le $5^{\circ}$ anniversaire de la SMCQ, il était d'emblée exclu de viser une présentation exhaustive de cet organisme qui est au cœur de la création musicale contemporaine au Québec depuis le 15 décembre 1966, date de son premier concert. Ses facettes sont beaucoup trop multiples, et l'espace manquerait rapidement. Toutefois, pour les lecteurs qui aimeraient tenir entre les mains un tel document, ils trouveront leur miel avec l'ouvrage de Réjean Beaucage paru au Septentrion en $2011^{2}$. $\mathrm{Si}$, par le détour d'une allusion à Salvador Dali, on comparait la SMCQ à une Vénus aux tiroirs, on pourrait dire que nous nous contenterons ici de n'ouvrir que quelques-uns de ces nombreux tiroirs. Évoquons dans un premier temps quelques-uns qui ne seront pas (ou peu) ouverts ici, mais qui, en revanche, ont déjà été abordés dans d'autres numéros de Circuit.

Lun des évènements les plus marquants de la SMCQ fut la Symphonie du millénaire (2000), œuvre collective de 19 compositeurs pour 333 musiciens et 2000 carillonneurs présentée à l'Oratoire Saint-Joseph devant environ 40000 personnes. C'est autour de cet évènement spectaculaire que tournait le numéro "Perceptions ${ }^{3}$ ». Ce numéro est, en effet, constitué de diverses "perceptions », à la fois de cet évènement en particulier et, plus généralement, de la musique contemporaine parmi la société. Du reste, ces deux axes de perception sont liés puisque la Symphonie du millénaire aspirait, justement, à briser une perception récurrente de déconnexion, de clivage, entre le milieu de la musique contemporaine et ledit «grand public». À cet égard, un débat avait d'ailleurs été déclenché au Québec à la suite d'une prise
1. Maryvonne Kendergi (1997), "Serge Garant, toujours présent", Circuit, musiques contemporaines, vol. 7, $\mathrm{n}^{\circ} 2$, p. 13.

2. Voir: www.septentrion.qc.ca/ catalogue/societe-de-musiquecontemporaine-du-quebec-la (consulté le 25 mai 2017). Soulignons au passage qu'un compte rendu de ce livre, rédigé par Éric Legendre, avait été publié dans les pages de Circuit en 2013 (vol. 23, $\mathrm{n}^{\circ} 2$, p. 84-87).

3. Vol. 11, nº 3, 2001. 
4. Vol. 7, $n^{\circ} 1,1996$.

5. Voir: www.smcq.qc.ca/mnm/fr/2017 (consulté le 27 mai 2017).

6. Vol. 14, $n^{\circ} 2,2004$.

7. Voir: www.smcq.qc.ca/smcq/fr/ hommage (consulté le 27 mai 2017).

8. Qui fut, d'ailleurs, brièvement directeur artistique de la SMCQ, entre 1986 et 1988 , assurant une forme d'intérim entre les années Garant et Boudreau.

9. Voir: www.revuecircuit.ca/ collection (consulté le 27 mai 2017).

10. Voir: www.smcq.qc.ca/jeunesse/fr (consulté le 27 mai 2017). de position de Lise Bissonnette, une discussion animée dont rend compte un numéro entier de Circuit, intitulé «Ruptures $?^{4} »$. Quant au tiroir du festival biennal Montréal/Nouvelles Musiques ( MNM $\left.^{5}\right)$, un numéro ${ }^{6}$ avait été consacré à sa toute première édition (en 2003), qui mettait les Pays-Bas à l'honneur. Soulignons que ces projets titanesques sont le fruit du tandem Walter Boudreau et Denys Bouliane, tandem qui a beaucoup fait au tournant des années 2000. Enfin, en ce qui concerne la Série hommage ${ }^{7}$, durant laquelle la SMCQ fédère, tous les deux ans (en alternance avec MNM) depuis 2007, une saison artistique entièrement focalisée sur un compositeur québécois important, nous nous référons à toutes ces monographies que nous pourrions baptiser la collection «Série hommage », consacrées successivement à Claude Vivier, Gilles Tremblay ${ }^{8}$, Ana Sokolović, Denis Gougeon, John Rea et, dès notre prochain numéro, José Evangelista9.

La SMCQ a toujours suscité le débat. Au départ, elle était le seul organisme voué à la musique contemporaine au Québec (il fut même le premier du genre au Canada). C'était alors une époque héroïque où une poignée de pionniers cherchaient à imposer leurs convictions, envers et contre tous. Avec le temps, l'organisme s'est - en toute conscience - institutionnalisé. En 2017, la SMCQ n’est pas spécialement la voie (ni la voix) de la jeunesse branchée et de l'underground, et le milieu des musiques de création s'est considérablement diversifié durant ce demi-siècle. Dès ce que nous pourrions appeler «l'ère Boudreau » (Walter Boudreau est directeur artistique depuis 1988), la SMCQ a cherché à se rapprocher d'un public le plus large possible. S'intéresser, par exemple, au minimalisme de Terry Riley, Steve Reich ou Louis Andriessen n'était pas nécessairement pour toujours plaire aux fondateurs, plus près de l'esprit moderniste européen de Darmstadt et des cours d'Olivier Messiaen au Conservatoire de Paris. Lorsqu'on lit les numéros de Circuit déjà mentionnés, consacrés à la Symphonie du millénaire ou à la première édition de MNM - numéros dirigés par Michel Duchesneau qui, rappelons-le, fut aussi directeur général de la SMCQ de 1997 à 2002 -, on trouve de nombreuses informations chiffrées, notamment sur les plans financiers, et des statistiques d'assistances. Cet angle vaut aussi pour le volet «Jeunesse » de la $\mathrm{SMCQ}^{10}$, qui s'est énormément développé depuis la fin des années 1990. Peut-être la prise de position de Lise Bissonnette, en 1994, marqua-t-elle une forme de tournant symbolique à cet égard. Cette polémique a suscité une vive indignation, certes, mais aussi une volonté ferme d'en finir avec cette perception (fondée ou pas, le débat reste ouvert, même plus de 20 ans plus tard) d'élitisme déconnecté. De fait, le milieu de la musique contemporaine a alors commencé à accélérer le pas dans la valse des communications et de 
la démocratisation culturelle. La direction artistique de Walter Boudreau se trouvait ainsi, dès le départ, entre l'arbre et l'écorce des esprits «purs et durs » d'une musique contemporaine se voulant radicale et sans compromis, et une approche plus conciliante et rassurante pour le «commun des mortels", dit-on. Le désir d'avoir une place dans les médias, par exemple, est dès lors devenu omniprésent.

Mais le débat a sans doute changé de direction - à un degré de profondeur et de durabilité qu'il est sans doute trop tôt pour commenter avec précision - dans la foulée du «Printemps érable» de 2012. Toute une génération de jeunes compositeurs sortant des écoles, voyant leurs aînés, souvent aigris, se débattre comme des diables pour presque rien, ont choisi de refuser de prime abord le paradigme dont ils étaient témoins. Plusieurs se sont orientés vers une approche moins affiliée aux institutions, en privilégiant le DIY (do it yourself) et l'idéal de liberté et d'indépendance qui l'accompagne (la contrepartie pouvant être, dans plusieurs cas, une usure rapide). Mais il y a d'autres enjeux aussi, à la croisée de l'esthétique et de l'éthique, liés notamment à la fin de l'hégémonie dominatrice de l'homme blanc et à une représentation plus diversifiée dans les programmes de concerts (enjeux touchant, donc, au féminisme et au multiculturalisme). Et, aussi, un refus drastique de ce qui s'apparente de près ou de loin au néolibéralisme. Plusieurs intellectuels, pas nécessairement toujours d'accord entre eux, nourrissent ces jeunes esprits contestataires: Alain Badiou, Normand Baillargeon, Judith Butler, Noam Chomsky, bell hooks et Slavoj Žižek, par exemple. Toujours est-il qu'en 2016, deux jeunes compositeurs ont enchaîné deux coups de gueule: Symon Henry, ici même dans les pages de Circuit, suivi de Gabriel Ledoux, sur son blogue personnel ${ }^{11}$. Réjean Beaucage, dans l'article qui ouvre ce numéro, évoque largement ce débat, qu'il contextualise face au passé de la SMCQ, et donne la parole à Walter Boudreau lui-même (un directeur artistique qui n’est pas né de la dernière pluie). Beaucage en profite pour, au passage, le questionner sur la programmation de la saison de la SMCQ soulignant son $50^{\mathrm{e}}$ anniversaire, saison marquée également par une édition du festival MNM sous le thème «Retour vers le futur ${ }^{12}$ » (dont le titre de ce numéro est une variation). Circuit a toujours fait de la place aux débats, y compris dans les numéros anniversaire ou dans les monographies, et nous ne dérogeons pas à ce principe (fondamentalement vivant) ici.

Parlant de «retour vers le futur», il est frappant combien le discours de certains compositeurs, notamment de la génération montante, revient à un état d'esprit proche, à certains égards, de celui des années 1950 à 1970. L'époque où il était de bon ton de balayer sans nuances Darmsdadt et les valeurs
11. Voir l'article de Réjean Beaucage, dans le présent numéro, pour davantage de détails sur ces prises de position.

12. Voir: www.smcq.qc.ca/mnm/ fr/2017 (consulté le 27 mai 2017). 
13. Voir, à cet égard, notre numéro "Souvenirs de Darmsdadt: retour sur la musique contemporaine du dernier demi-siècle" (vol. 15, nº 3, 2005).

14. Vol. $7, n^{0} 2,1996$

15. Louise Bail, Maryvonne Kendergi: musique en partage, Montréal, Hurtubise, 2002. modernistes du revers de la main est peut-être révolue ${ }^{13}$ (ceux qui parlent de retour du pendule sont peut-être, en ce sens, un retour en retard). Les années héroïques des débuts de la SMCQ - l’ère Garant - suscitent chez beaucoup de jeunes (et moins jeunes) compositeurs un regain d'intérêt marqué. Dans ce contexte, nous recommandons bien sûr à nos lecteurs de (re)lire le numéro qui fut consacré à Serge Garant ${ }^{14}$. Dans le cadre du présent opus, c'est plutôt vers Maryvonne Kendergi que nous avons souhaité nous tourner pour relater cette époque. En effet, les débuts de la SMCQ étaient particulièrement internationalistes, et d'aucuns lui ont même reproché, dans cette perspective, un certain colonialisme (à tout le moins un mimétisme des grands courants européens). Quoiqu'il en soit, cette génération était très ouverte sur le monde et en phase avec les recherches les plus actuelles de l'époque. Maryvonne Kendergi a joué, à ce titre, un rôle plus que considérable, et c'est pourquoi nous avons demandé à Louise Bail - auteure d'un livre important sur cette femme d'exception ${ }^{15}$ - d'écrire un article sur l'apport de Kendergi à la SMCQ, tout particulièrement du point de vue des liens internationaux.

Il nous importait, dans ce numéro sur la SMCQ, de ne pas ouvrir mille et une discussions d'ordre social et sociétal sur cet organisme sans même parler de ce qu'il fait artistiquement. Le spectacle La vie qui bat (1999), une coproduction de la SMCQ et de la compagnie de danse $O$ Vertigo, nous a paru à cet égard pertinent sur plusieurs plans. Par exemple, sa musique - issue de Drumming (1971) de Steve Reich - est de celles qu’a défendues Boudreau durant son «règne», ce qui ne fit pas nécessairement plaisir à tous. Par ailleurs, il s'agit d'une coproduction, et la SMCQ a, justement, beaucoup misé sur les partenariats depuis l'ère Boudreau. Enfin, c'est là une rencontre multidisciplinaire, avec la danse qui plus est, un art qui a beaucoup de succès au Québec et dont la musique contemporaine envie souvent la place importante qu'elle occupe, tant localement qu' internationalement. Ce spectacle fait ici l'objet d'une étude signée par la percussionniste Aiyun Huang. Lun des intérêts de cet article est, notamment, la mise en contexte internationale de cette production significative dans l'histoire de la SMCQ.

Un autre exemple absolument crucial de ce que fait la SMCQ (et il est parfois sujet à débat de se demander si elle le fait encore assez) est la commande (et/ou la création) de nouvelles œuvres. Il était très intéressant, en ce sens, de demander à Solenn Hellégouarch, notre directrice administrative et secrétaire de rédaction, de dresser un catalogue des œuvres commandées (ou co-commandées) et créées par la SMCQ. Parcourir cette liste est très instructif, et nous laissons au lecteur le soin de faire ses propres observations. Le Cahier d'analyse, signé Michel Gonneville, procède pour sa part à une 
sorte de «zoom avant» sur l'une de ces créations (qu'il passe au microscope), à savoir Circuits parallèles de Nicolas Gilbert. Outre le titre de cette œuvre qui, bien sûr, ne manque pas de nous plaire, il nous paraissait intéressant que Gonneville écrive sur Gilbert, l'inverse s'étant produit en 2009, alors que ce dernier offrait dans nos pages une analyse de Chute/Parachute, un «classique» de Gonneville ${ }^{16}$. Rappelons que Circuits parallèles, créée le 24 avril $2008^{17}$ par l'Ensemble de la SMCQ, avait ensuite mérité le prix Opus de la Création de l'année, remis par le Conseil québécois de la musique.

Quant aux illustrations du numéro, exceptionnellement nombreuses, nous avons opté pour une sorte de «scrapbook» relatant en images les 50 ans d'histoire de la société par autant de photographies d'archives. Celles-ci peuvent également être visionnées en ligne, sur notre site $\mathrm{Web}^{18}$. J'en profite pour remercier au passage la SMCQ - en particulier Aïda Aoun, Walter Boudreau, Jacques Cabana et Noémie Pascal - dont l'esprit de collaboration pour ce numéro a été exemplaire à tous égards, ainsi que la Fondation SOCAN, dont une aide financière spéciale nous permet un numéro un peu plus volumineux que d'habitude.

Enfin, en complément au dossier thématique, notre rubrique Actualités propose deux comptes rendus. Jimmie LeBlanc présente un ouvrage collectif (sous la direction de Jean-Marc Chouvel et Pascale Criton) sur la "penséemusique» du philosophe Gilles Deleuze, tandis que Nicolas-Alexandre Marcotte relate Björk Digital, une exposition présentée récemment en première américaine par le Centre Phi et DHC/ART, à Montréal.

En terminant, c'est avec enthousiasme et fierté que nous annonçons à nos lecteurs la constitution, à Circuit, d'un tout nouveau comité externe qui viendra appuyer le comité de rédaction. Ce comité externe, à la fois interdisciplinaire et international, est constitué de Lucie Bourassa, Pascale Criton, Jonathan Cross, Valérie Dufour, Christoph Haffter et Jean-Noël von der Weid.

Bonne lecture!

Montréal, mai 2017

\section{Addendum}

Au moment de mettre sous presse, nous apprenions avec tristesse le décès de l'immense compositeur, pédagogue et penseur Gilles Tremblay (1932-2017), à qui Circuit a déjà consacré pas moins de trois numéros. Les trajectoires de ce créateur et de la SMCQ sont, d'ailleurs, étroitement liées. Et c'est un bel hasard qu'il apparaisse dès l'ouverture de ce numéro, en compagnie de Walter Boudreau, dans un état évident de jubilation «alleluiesque», ce qui était peut-être sa signature la plus profonde. Nous dédions ce numéro à sa mémoire.
16. Nicolas Gilbert, "Chute/Parachute de Michel Gonneville: pensée sérielle, écriture postmoderne (ou l'inverse)", Circuit, musiques contemporaines, vol. 19, $\mathrm{n}^{\circ} 3$, 2009, p. 91-108.

17. À noter que Gilbert, né en 1979 , n'avait alors que 28 ans. Peu de compositeurs dans la vingtaine ont reçu des commandes de la SMCQ.

18. www.revuecircuit.ca (consulté le 27 mai 2017). 